\title{
Polycystic ovary syndrome and mental disorders: a systematic review and exploratory meta-analysis
}

This article was published in the following Dove Press journal:

Neuropsychiatric Disease and Treatment

8 November 2016

Number of times this article has been viewed

\author{
Sergio Luís Blay' \\ João Vicente Augusto \\ Aguiar ${ }^{2}$ \\ Ives Cavalcante Passos ${ }^{3}$ \\ 'Department of Psychiatry, Federal \\ University of São Paulo (Escola \\ Paulista de Medicina - UNIFESP), \\ São Paulo, SP, Brazil; ${ }^{2}$ Department \\ of Psychiatry, Fortaleza University, \\ Fortaleza, Ceará, Brazil; 'ंLaboratory \\ of Molecular Psychiatry and \\ Department of Psychiatry, Federal \\ University of Rio Grande do Sul, \\ Rio Grande do Sul, Brazil
}

Background: The association between depression, anxiety, and polycystic ovary syndrome (PCOS) is still unclear. Therefore, a systematic review and meta-analysis was conducted to assess the rates of comorbid psychiatric disorders among women with PCOS compared to women without it.

Methods: PubMed/MEDLINE, Embase, PsycINFO, and Web of Science databases were searched from inception to November 27, 2015. Studies were eligible for inclusion if they were original reports in which the rates of mood (bipolar disorder, dysthymia, or major depressive disorder), obsessive-compulsive spectrum disorders, trauma- and stressor-related disorders, anxiety disorders or psychotic disorders, somatic symptom and related disorders, or eating disorders had been investigated among women with an established diagnosis of PCOS and compared with women without PCOS. Psychiatric diagnosis should have been established by means of a structured diagnostic interview or through a validated screening tool. Data were extracted and pooled using random effects models.

Results: Six studies were included in the meta-analysis; of these, five reported the rates of anxiety and six provided data on the rates of depression. The rate of subjects with anxiety symptoms was higher in patients with PCOS compared to women without PCOS (odds ratio $(\mathrm{OR})=2.76 ; 95 \%$ confidence interval $(\mathrm{CI}) 1.26$ to 6.02 ; $\log \mathrm{OR}=1.013 ; P=0.011$ ). The rate of subjects with depressive symptoms was higher in patients with PCOS compared to women without PCOS (OR $=3.51 ; 95 \%$ CI 1.97 to 6.24 ; $\log \mathrm{OR}=1.255 ; P<0.001)$.

Conclusion: Anxiety and depression symptoms are more prevalent in patients with PCOS.

Keywords: polycystic ovary syndrome, depression, anxiety, epidemiological survey, metaanalysis, systematic review

\section{Introduction}

Polycystic ovary syndrome (PCOS) is a complex genetic disease that affects approximately $7 \%$ of women of reproductive age worldwide. ${ }^{1}$ PCOS is a heterogeneous disorder, where the main clinical features include menstrual irregularities, subfertility, hyperandrogenism, and hirsutism. ${ }^{2}$

Numerous studies have evaluated the relation between PCOS and psychiatric disorders; however, most have evaluated psychiatric symptoms based on self-report measures. ${ }^{1,3}$ There remains, therefore, an unclear relationship between PCOS and psychiatric disorders. Cross-sectional epidemiological studies have reported that individuals with PCOS are more likely to have anxiety or depressive disorders when compared to those in the general population. ${ }^{4}$ Two studies have shown depression, bipolar disorder, anxiety disorders, and binge eating disorder are more frequent among women with PCOS compared with controls. ${ }^{5,6}$
Correspondence: Sergio Luís Blay Department of Psychiatry - UNIFESP, R. Borges Lagoa, 570 CEP 04038-020 São Paulo, SP, Brazil Tel/fax +55 II 38161030

Email blay@uol.com.br 
PCOS is accompanied by an array of symptoms such as obesity, acne, scalp hair thinning, menstrual irregularity, and subfertility. These symptoms can also contribute to psychological impairment. ${ }^{7}$ Despite the use of antidepressants in the treatment of depressive symptoms, there has been only one report to date on the use of serotonin reuptake inhibitors with a successful outcome in a patient with PCOS. ${ }^{8}$

In light of the above mentioned, the purpose of the present study is to perform a systematic review and metaanalysis of the rates of mood (bipolar disorder, dysthymia, or major depressive disorder), obsessive-compulsive spectrum disorders, trauma- and stressor-related disorders, anxiety disorders, psychotic disorders, somatic symptom and related disorders, binge eating disorders and eating disorders among women with an established diagnosis of PCOS compared to those without PCOS.

\section{Materials and methods}

A systematic review and meta-analysis was performed in accordance with the Preferred Reporting Items for Systematic Reviews and Meta-Analyses (PRISMA) and the Cochrane group guideline recommendations. ${ }^{9}, 10$ The process includes literature review, eligibility criteria of the retrieved references, assessment of the methodological quality of included studies, extraction of outcomes and relevant variables, and meta-analysis of the data.

\section{Search strategy}

We searched the PubMed/MEDLINE, Embase, PsycINFO, and Web of Science databases from inception to November 27 , 2015. This search strategy was augmented by hand-searching through reference lists of included articles and by tracking the citations of eligible references in Google Scholar.

\section{Eligibility criteria}

We included original reports in which the rates of mood (bipolar disorder, dysthymia, or major depressive disorder), obsessive-compulsive spectrum disorders, trauma- and stressor-related disorders, anxiety disorders, psychotic disorders, somatic symptom and related disorders, and eating disorders have been investigated among women with an established diagnosis of PCOS and compared to women without PCOS. Psychiatric diagnosis should have been established by means of a structured diagnostic interview according to either Diagnostic and Statistical Manual of Mental Disorders (DSM) or International Classification of Diseases (ICD) criteria. Studies in which a diagnosis of psychiatric disorder had been established through a validated screening tool above a preestablished cutoff were also considered for inclusion. A diagnosis of PCOS could be established by means of the Rotterdam, National Institutes of Health (NIH), or PCOS Society criteria. ${ }^{11}$ We included original peer-reviewed studies published in English, Portuguese, French, Spanish, or German. Either populationbased or studies performed in clinical samples were eligible for inclusion. Meeting abstracts were excluded. We also excluded studies performed in pediatric populations.

\section{Outcomes}

The primary outcome was the odds ratios (ORs) of psychiatric disorders among women with PCOS compared to women without PCOS.

\section{Search strategy (November 27, 20I5) PubMed/MEDLINE}

- Search 1: (()(()((“Depression”[Mesh] OR “Depressive Disorder”[Mesh] OR “Depression, Postpartum”[Mesh] OR “Depressive Disorder, Major”[Mesh] OR "Bipolar Disorder”[Mesh]) OR “Anxiety Disorders”[Mesh]) OR "Phobic Disorders"[Mesh]) OR “Stress Disorders, Post-Traumatic"[Mesh]) OR "Obsessive-Compulsive Disorder"[Mesh]) OR "Psychotic Disorders"[Mesh]) OR ("Schizophrenia"[Mesh] OR "Schizophrenia and Disorders with Psychotic Features"[Mesh])) OR "Somatoform Disorders"[Mesh]) OR ("Eating Disorders”[Mesh] OR “Binge Eating Disorder”[Mesh]) Field: Title/Abstract.

- Search 2: "Polycystic Ovary Syndrome"[Mesh] Field: Title/Abstract.

- Search 3: \#1 AND \#2.

- Retrieved references: 171.

\section{OVID databases (PsycINFO and Embase Classic plus}

\section{Embase)}

- Search 1: "polycystic ovary syndrome".ti,ab,kw

- Search 2: ("depression" or "depressive disorder" or "major depressive disorder" or "bipolar disorder" or "mania" or "postpartum depression" or "somatoform disorder" or "binge eating disorder" or "eating disorder" or "anxiety disorder" or "post-traumatic stress disorder" or "obsessive compulsive disorder" or "psychosis" or "psychotic disorder" or "schizophrenia" or "bulimia" or "anorexia nervosa").

- Search 3: \#1 and \#2.

- Retrieved References: 360. 


\section{Selection of eligible studies}

- This systematic review complied with PRISMA guidelines;

- the primary (ie, title/abstract) screening was independently performed by two researchers (SLB and JVAA), supervised by ICP, who made the final decision in cases of disagreement;

- the PDFs (ie, full-texts) of potentially eligible articles were retrieved;

- the secondary screening was independently performed by two researchers (SLB and JVAA), supervised by ICP, who made the final decision in cases of disagreement;

- disagreements were resolved through consensus.

\section{Quality assessment}

We used the Newcastle-Ottawa Quality Assessment Scale (NOQAS) to assess the quality of included studies. ${ }^{12}$ Overall quality score was defined as the percentage of criteria that were met by each included study. We excluded NOQAS items 4 and 7 because these were meaningless for the context of the current study. The agreement of independent raters (SLB and JVAA) was excellent (Cohen's kappa $=0.91$; standard error $(\mathrm{SE})=0.05)$.

\section{Data extraction}

We built Excel spreadsheets to extract data from included articles. We used the version X7 of EndNote to remove duplicate data. The characteristics extracted from each study were: name of the first author; publication year; country; number, sex, and age of the patients and controls; type of diagnostic criteria used to assess PCOS (Rotterdam, NIH, or PCOS Society) and the psychiatric disorders (DSM or ICD); whether patients were medication free; latitude of the city where the study was performed; mean age of the total sample size; type of study (population-based or clinical study); whether the diagnoses of psychiatric disorders were assessed through structured interviews; NOQAS items.

\section{Data analysis}

We used the metaphor package for $\mathrm{R}$ (version number 3.2.3) to do the meta-analysis with binary data and meta-regression analysis. ${ }^{13}$ We undertook meta-analyses whenever values of the rate of a specific psychiatric disorder in patients with PCOS compared to healthy controls were available in two or more studies. ${ }^{13} \mathrm{~A}$ random-effects model with restricted maximum-likelihood estimator was used to synthesize the effect size across studies. This model can incorporate both within-study variability and between-study variability. ${ }^{13}$
The OR and Log OR were used to assess the effect size. ${ }^{8}$ The significance level for this meta-analysis model was $0.05 .{ }^{13}$ Egger's linear regression test was used to assess publication bias. This is a test for asymmetry of the funnel plot, and it was done whenever three or more studies were included..$^{14}$ For this specific test a $P$-value of less than 0.1 shows significant asymmetry and therefore publication bias. ${ }^{14}$ If Egger's linear regression test revealed a potential publication bias, we used Duval and Tweedie's trim and fill method to test the data. We also used the so-called leave-one-out function for doing sensitivity analysis. ${ }^{13}$ This method consists of the removal of one study at a time from the dataset to run the meta-analysis without it. This analysis tests if the effect size of the metaanalysis is driven by one study. We used the $Q$ statistic to test the existence of heterogeneity and $I^{2}$ to assess the proportion of total variability due to heterogeneity. ${ }^{15}$ An $I^{2}$ value of $\sim 25 \%$ could be regarded as low, $\sim 50 \%$ as medium, and $\sim 75 \%$ as high. ${ }^{9}$ We used $\tau^{2}$ to estimate the total amount of heterogeneity. ${ }^{15}$

We explored sources of heterogeneity in studies using univariate meta-regression analysis. ${ }^{16,17} \mathrm{We}$ selected the latitude of the city where the study was performed, type of diagnostic criteria used to define PCOS, mean age of the total sample size of the included studies, type of study, total sample size of the PCOS group of the included studies, whether the diagnoses of psychiatric disorders were assessed through structured interviews, and quality of the included studies as assessed by the NOQAS as moderator. A pseudo- $R^{2}$ statistic is given for each analysis, showing the percentage of total heterogeneity in the true effects that is accounted for by the model with all covariates included.

\section{Results}

Figure 1 shows the study selection process. Quality and characteristics of included studies are described in Table 1.

\section{Anxiety symptoms}

A total of five studies were included in this meta-analysis. ${ }^{18-22}$ The rate of subjects with anxiety symptoms was higher in patients with PCOS compared to healthy controls $(\mathrm{OR}=2.76$; 95\% confidence interval (CI) 1.26 to 6.02 ; $\log \mathrm{OR}=1.013$; $P=0.011)$. Figure 2 shows the forest plot and the $\mathrm{OR}$ of the pooled analysis (Log scale was used in the $\mathrm{x}$ axis) while Figure 3 shows the funnel plot (Log OR was used for the funnel plot). Egger's linear regression test revealed potential publication bias $(z=1.869, P=0.061)$. We, therefore, performed Duval and Tweedie's trim and fill method, and the result for the rate of anxiety symptoms in patients with PCOS was still 


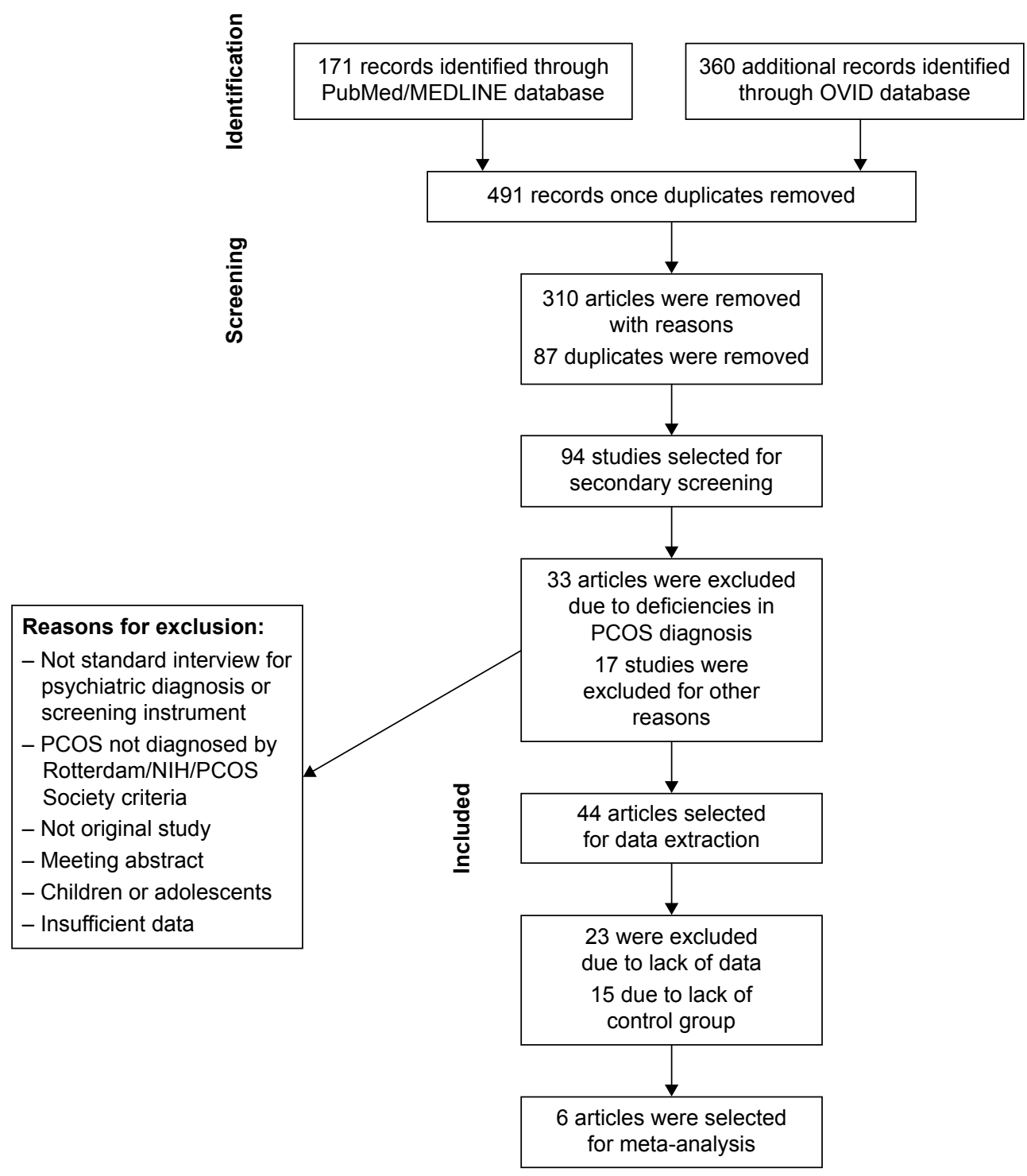

Figure I PRISMA flowchart of systematic review.

Abbreviations: PCOS, polycystic ovary syndrome; $\mathrm{NIH}$, National Institutes of Health.

higher compared to healthy controls $(\mathrm{OR}=2.40 ; 95 \% \mathrm{CI} 1.16$ to $4.97 ; \log \mathrm{OR}=0.874 ; P=0.018$ - one study was estimated on the left side). The significance of the effect size remained robust when leave-one-out models were used for such metaanalysis (see Table 2 - results were presented with Log OR). No significant heterogeneity was found $(Q$ statistic $=8.345$, $\left.d f=4, P=0.079 ; I^{2}=51.73 \% ; \tau^{2}=0.389\right)$. In our investigation of covariates using univariate meta-regression analyses, we found that latitude of the city where the study was performed (estimate $=0.010 ; 95 \% \mathrm{CI}-0.013$ to $0.034 ; P=0.375$ ), type of diagnostic criteria used to define PCOS (estimate $=0.393$; $95 \%$ CI -1.452 to $2.240 ; P=0.675$ ), mean age of the total sample size of the included studies (estimate $=-0.123 ; 95 \%$ $\mathrm{CI}-0.264$ to $0.017 ; P=0.085)$, type of study (estimate $=0.393$;
$95 \% \mathrm{CI}-1.452$ to $2.240 ; P=0.675)$, total sample size of the PCOS group of the included studies (estimate $=0.012 ; 95 \%$ $\mathrm{CI}-0.032$ to $0.056 ; P=0.597$ ) and whether the diagnoses of psychiatric disorders were assessed through structured interviews (estimate $=-1.307 ; 95 \% \mathrm{CI}-3.848$ to 1.233 ; $P=0.313$ ) did not moderate the effect size. It is worth mentioning that quality of the included studies as assessed by the Newcastle-Ottawa Scale also did not moderate the effect size (estimate $=-0.711 ; 95 \% \mathrm{CI}-1.317$ to $-0.104 ; P=0.021$ ).

\section{Depressive symptoms}

A total of six studies were included in this metaanalysis. ${ }^{18-20,22,23}$ The rate of subjects with depressive symptoms was higher in patients with PCOS compared 


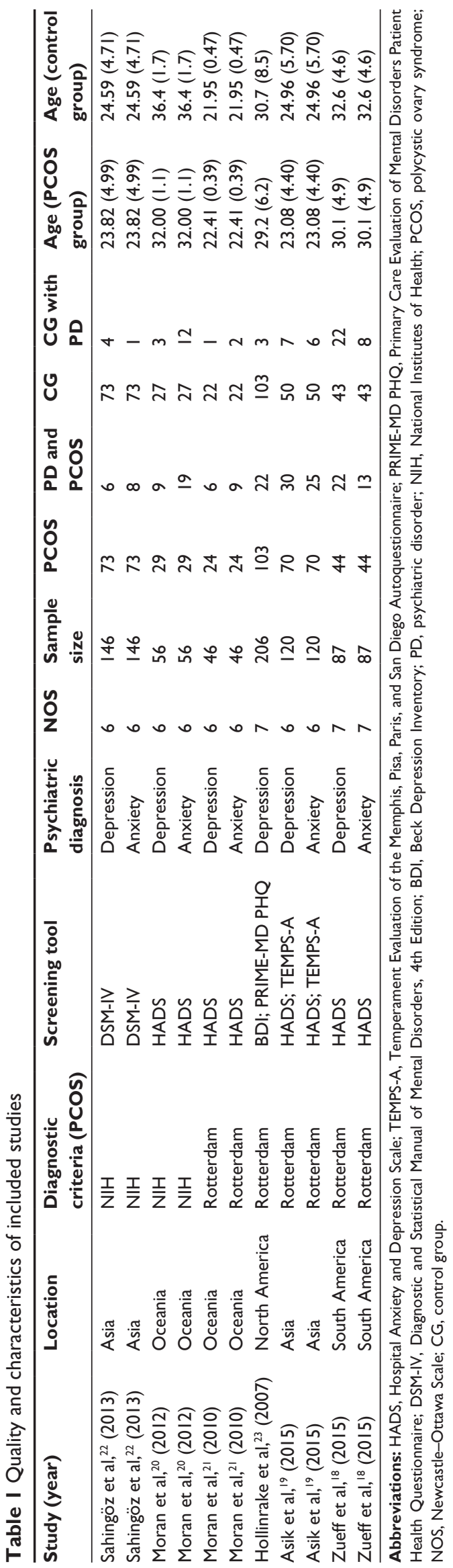

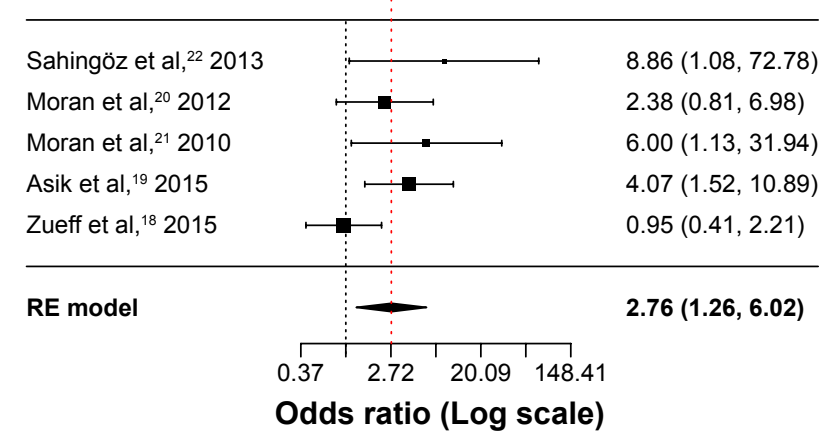

Figure 2 Forest plot of the studies assessing anxiety symptoms. Abbreviation: RE model, random-effects model.

to healthy controls (OR $=3.51 ; 95 \%$ CI 1.97 to 6.24 ; $\log$ $\mathrm{OR}=1.255 ; P<0.001)$. Figure 4 shows the forest plot and the OR of the pooled analysis (Log scale was used in the $\mathrm{x}$ axis) while Figure 5 shows the funnel plot (Log OR was used for the funnel plot). Egger's linear regression test revealed no potential publication bias $(z=0.499, P=0.618)$. The significance of the effect size remained robust when leave-one-out models were used for such meta-analysis (see Table 3 - results were presented with Log OR). We found no significant between-study heterogeneity $(Q$ statistic $=6.055$, $\left.d f=5, P=0.301 ; I^{2}=22.17 \% ; \tau^{2}=0.338\right)$. In our investigation of covariates using univariate meta-regression analyses, we found the city's latitude where the study was performed (estimate $=0.005 ; 95 \% \mathrm{CI}-0.012$ to $0.022 ; P=0.551$ ), type of diagnostic criteria used to define PCOS (estimate $=-0.606$; 95\% CI -1.926 to $0.713 ; P=0.367$ ), mean age of the total sample size of the included studies (estimate $=-0.069 ; 95 \%$ CI -0.201 to $0.061 ; P=0.298$ ), type of study (estimate $=-0.606$; $95 \% \mathrm{CI}-1.926$ to $0.713 ; P=0.367)$, total sample size of the PCOS group of the included studies (estimate $=0.011 ; 95 \%$ CI -0.009 to $0.032 ; P=0.284$ ), and whether the diagnoses of psychiatric disorders were assessed through structured interviews (estimate $=-0.531 ; 95 \% \mathrm{CI}-3.485$ to 2.422 ;

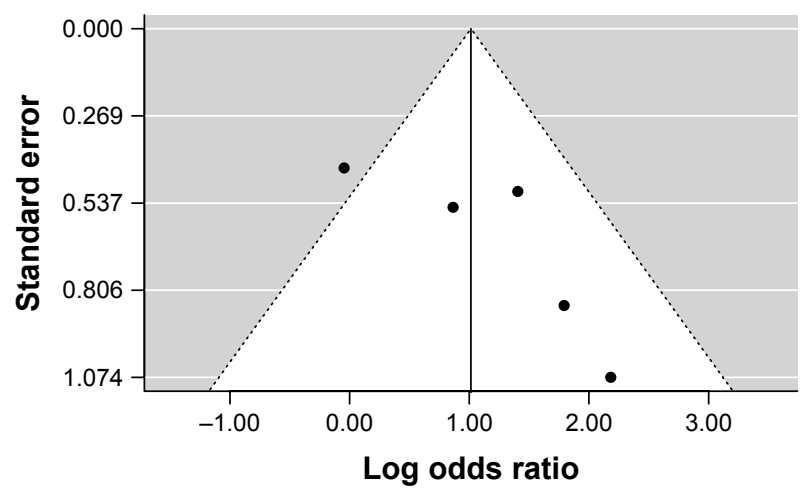

Figure 3 Funnel plot of the studies that assessed anxiety symptoms. 
Table 2 Leave-one-out models for the meta-analysis of anxiety symptoms

\begin{tabular}{|c|c|c|c|c|c|c|c|c|}
\hline \multirow[t]{2}{*}{ Study (year) } & \multirow{2}{*}{$\begin{array}{l}\text { Log odds } \\
\text { ratio }\end{array}$} & \multirow[t]{2}{*}{ SE } & \multirow[t]{2}{*}{$z$ value } & \multirow[t]{2}{*}{$P$-value } & \multirow[t]{2}{*}{$95 \% \mathrm{Cl}$} & \multicolumn{3}{|l|}{ Heterogeneity } \\
\hline & & & & & & $Q$ statistic ( $P$-value) & $\tau^{2}$ & $I^{2}$ \\
\hline Sahingöz et al, ${ }^{22}(2013)$ & 0.874 & 0.410 & 2.128 & $P=0.033$ & 0.069 to 1.679 & $6.686(P=0.082)$ & 0.357 & $54.2 \%$ \\
\hline Moran et al, ${ }^{20}(2012)$ & 1.119 & 0.530 & 2.111 & $P=0.034$ & 0.080 to 2.158 & $8.342(P=0.039)$ & 0.658 & $62.3 \%$ \\
\hline Moran et al, ${ }^{21}(2010)$ & 0.887 & 0.435 & 2.039 & $P=0.04 \mathrm{I}$ & 0.034 to 1.739 & $6.970(P=0.072)$ & 0.403 & $55.8 \%$ \\
\hline Asik et al, ${ }^{19}(2015)$ & 0.925 & 0.500 & 1.849 & $P=0.064$ & -0.055 to 1.906 & $6.610(P=0.085)$ & 0.529 & $55.4 \%$ \\
\hline Zueff et al, ${ }^{18}(2015)$ & 1.343 & 0.324 & 4.146 & $P<0.001$ & 0.708 to 1.979 & I.656 $(P=0.646)$ & 0 & $0 \%$ \\
\hline
\end{tabular}

Abbreviations: SE, standard error; $\mathrm{Cl}$, confidence interval.

$P=0.724$ ) did not moderate the effect size. It is worth mentioning that quality of the included studies as assessed by the Newcastle-Ottawa Scale also did not moderate the effect size (estimate $=-0.131 ; 95 \% \mathrm{CI}-0.929$ to $0.667 ; P=0.747$ ). We did not analyze structured psychiatric diagnosis as a moderator since no study used it.

\section{Discussion}

This systematic review found six studies that study the association of PCOS with depression or anxiety in young women (22.4 to 32 years old in the PCOS group and 24.9 to 36.4 years old in the control group) in clinical settings, performed in four countries (Turkey, Australia, Brazil, United States). Methodologically, all studies selected were performed in clinical settings, with a good overall assessment of quality, although they showed some different characteristics, such as sample size (between 46 and 206 individuals), differences in the criteria used to diagnose PCOS as well as a difference in the instruments used to assess anxiety and/or depression. Particularly, the lack of a unified concept of PCOS and differences in the assessment of psychiatric diagnosis across studies hindered comparisons. However, we were not able to identify potential sources of heterogeneity (latitude, type of diagnostic criteria used to define PCOS, mean age, type of study, total sample size of the PCOS group, assessment of psychiatric disorders, and methodological quality of the study).

\begin{tabular}{|c|c|}
\hline Sahingöz et al, ${ }^{22} 2013$ & $1.54(0.42,5.72)$ \\
\hline Moran et al, ${ }^{20} 2012$ & $3.60(0.86,15.12)$ \\
\hline Moran et al, ${ }^{21} 2010$ & $7.00(0.77,63.72)$ \\
\hline Hollinrake et al, ${ }^{23} 2007$ & $9.05(2.62,31.33)$ \\
\hline Asik et al,,$^{19} 2015$ & $4.61(1.82,11.66)$ \\
\hline Zueff et al, ${ }^{18} 2015$ & $1.83(0.67,5.01)$ \\
\hline RE model & $3.51(1.97,6.24)$ \\
\hline 0.37 & 148.41 \\
\hline & \\
\hline
\end{tabular}

Figure 4 Forest plot of the studies that assessed depressive symptoms. Abbreviation: RE model, random-effects model.
These results should be considered exploratory in nature as few studies may undermine the statistical power of metaregression techniques.

Overall, in the present meta-analysis, we found that rates of anxiety and depressive symptoms were higher in patients with PCOS compared to healthy controls in line with distinct clinical studies suggesting the increased prevalence of anxiety and depression in women with PCOS.

\section{Clinical and research implications}

Results from this systematic review indicate that specialized literature shows that a limited number of studies have assessed the prevalence of psychiatric conditions in women with PCOS compared to healthy controls. Only six studies were selected. The main finding was that women with PCOS have a higher prevalence rate of anxiety and depression.

Potential mechanisms for these associations may include social, psychological, and neurobiological aspects. Women with PCOS may report clinically significant symptoms of anxiety or depression at least in part as a result of significant body changes imposed by their illness (eg, hirsutism, irregular menses, obesity, acne, hair thinning). ${ }^{2,7}$ Previous research indicates that alterations in body image may contribute to psychological distress among women with PCOS. ${ }^{24}$

The literature on neurobiological research in anxiety disorders, anxiety symptoms, depressive disorders, and depressive symptoms among women with PCOS

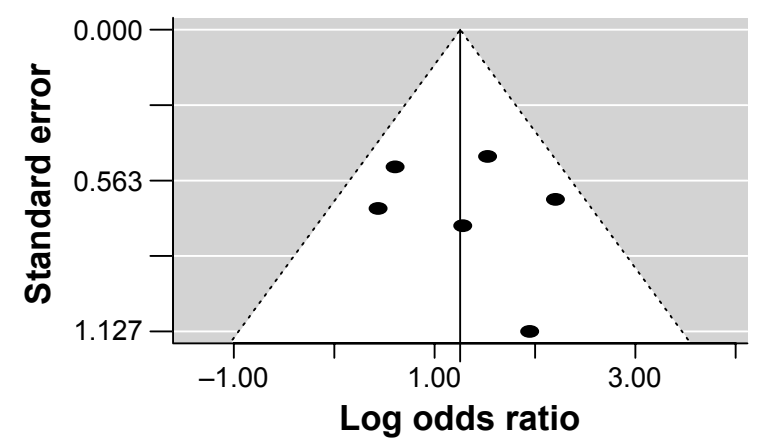

Figure $\mathbf{5}$ Funnel plot of the studies that assessed depressive symptoms. 
Table 3 Leave-one-out models for the meta-analysis of depressive symptoms

\begin{tabular}{|c|c|c|c|c|c|c|c|c|}
\hline \multirow{2}{*}{$\begin{array}{l}\text { Excluded } \\
\text { study (year) }\end{array}$} & \multirow{2}{*}{$\begin{array}{l}\text { Log odds } \\
\text { ratio }\end{array}$} & \multirow[t]{2}{*}{ SE } & \multirow[t]{2}{*}{$z$ value } & \multirow[t]{2}{*}{$P$-value } & \multirow[t]{2}{*}{$95 \% \mathrm{Cl}$} & \multicolumn{3}{|l|}{ Heterogeneity } \\
\hline & & & & & & Q statistic ( $P$-value) & $\tau^{2}$ & $P^{2}$ \\
\hline Sahingöz et al, ${ }^{22}(2013)$ & 1.401 & 0.313 & 4.477 & $P<0.00$ I & 0.788 to 2.015 & $4.33 \mathrm{I}(P=0.363)$ & 0.097 & $19.6 \%$ \\
\hline Moran et al, ${ }^{20}(2012)$ & 1.258 & 0.349 & 3.598 & $P<0.001$ & 0.572 to 1.943 & $6.052(P=0.195)$ & 0.215 & $35.9 \%$ \\
\hline Moran et al, ${ }^{21}(2010)$ & 1.209 & 0.312 & 3.864 & $P<0.001$ & 0.595 to 1.822 & $5.649(P=0.226)$ & 0.142 & $29.1 \%$ \\
\hline Hollinrake et al, ${ }^{23}$ (2007) & 1.065 & 0.276 & 3.851 & $P<0.001$ & 0.523 to 1.607 & $3.340(P=0.502)$ & 0.002 & $0.4 \%$ \\
\hline Asik et al, ${ }^{19}(2015)$ & 1.179 & 0.375 & 3.144 & $P=0.001$ & 0.444 to 1.914 & $5.562(P=0.234)$ & 0.225 & $32.4 \%$ \\
\hline Zueff et al, ${ }^{18}(2015)$ & 1.451 & 0.295 & 4.914 & $P<0.001$ & 0.872 to 2.030 & $3.997(P=0.406)$ & 0.011 & $2.4 \%$ \\
\hline
\end{tabular}

Abbreviations: $\mathrm{SE}$, standard error; $\mathrm{Cl}$, confidence interval.

encompasses hypothalamic-pituitary-adrenal (HPA) axis activity and neuroimaging studies. ${ }^{25}$

The neurophysiologic etiology of anxiety and depression is not fully understood but the dysregulation of the HPA axis has been linked to stress and, although less extensively, its putative association with anxiety and depression disorders has also been studied. ${ }^{26}$

Higher basal cortisol levels have been described in depression, panic disorder, and generalized anxiety disorders. In the latter condition, studies suggest that such dysregulation may be state dependent, as higher cortisol levels can be found in persons with current anxiety disorder and borderline cortisol levels for those with remitted symptoms ${ }^{27}$ The maintenance of basal HPA tone is essential for homeostasis, which is usually disrupted in depressive and PCOS patients. ${ }^{25,26,28-30}$ There is evidence showing women with PCOS have several HPA changes such as abnormal androgen synthesis and secretion, enhanced peripheral metabolism of cortisol, ${ }^{31}$ compensatory overdrive of HPA, ${ }^{10}$ and impaired feedback inhibition is likely in patients with major depression. ${ }^{11,12}$ These changes might explain some of the underlying pathophysiological mechanisms of the association between PCOS and depression.

Through imaging studies there is a body of evidence suggesting that lateral and medial regions of the prefrontal cortex (PFC) modulate amygdala and related limbic structures during effortful emotion regulation. Anxiety disorders may be linked to an imbalance between the PFC and the amygdala. Marsh et al assessed mood, metabolic function, and neuronal activation during an emotional task through functional magnetic resonance imaging, and $\mu$-opioid receptor availability using positron emission tomography within PCOS patients. Major findings indicated patients with PCOS had significantly greater activity in the PFC and ventral anterior cingulate cortex compared to controls. ${ }^{25}$

\section{Clinical and research impact}

Women with more serious PCOS symptoms are probably more likely to get treatment from their gynecologists.
It should be emphasized that consultation is an opportunity to assess not only the clinical aspects of PCOS but also the clinical symptoms of anxiety or depression that could be effectively treated. In addition, it is a chance to address concerns that are not necessarily associated with a psychiatric condition or a psychiatric diagnosis (eg, marital, family, social issues, low level of quality of life, sexual dysfunction, low self-esteem) that often could be PCOS associated.

Given the seriousness of anxiety and depression conditions in women with PCOS, further study of epidemiology, clinical features, neurobiology, disability, quality of life, and treatment in different settings and countries is needed to better understand this association.

Two other reviews analyzing the relation between depression and anxiety and PCOS were found. Both, Barry et al and Veltman-Verhulst et al arrived at similar conclusions found in this study, even though the number of articles investigated in those studies differed mainly due to different inclusion and exclusion criteria. ${ }^{32,33}$

\section{Conclusion}

Results from this systematic review indicate that specialized literature shows a lack of data on the association of PCOS and mental disorders. Only six studies were selected and even these used heterogeneous concepts of assessment.

Individuals with PCOS showed a greater prevalence of anxiety and depressive symptoms. New investigations could focus on establishing standard assessment procedures as well as undertaking a cost-benefit assessment to establish whether treating anxiety and depression in individuals with PCOS could lead to better directives on the treatment of these patients.

\section{Limitations}

This systematic review is characterized by the following limitations: (1) the search for studies eligible for inclusion was performed using electronic databases and only studies published in specialized literature, rather than in books, textbooks, or theses, were selected; (2) according to the criteria for inclusion in this 
review, the selection of languages could have excluded studies performed in other countries or continents; (3) a reduced number of studies was included, which restricted the performance of a meta-analysis; (4) the instruments to assess anxiety or depression were different across studies; (5) the NOQAS for assessing the methodological quality of each study was adapted for the purposes of our analysis; however, inter-rater reliability of the scale was kept appropriate; (6) the use of obese patients ${ }^{16}$ as a control group in one study could affect the results of this review. However, sensitivity analysis did not show that such a control group affected the study conclusions.

\section{Suggestions for future research}

New studies on the prevalence of anxiety and depression in individuals with PCOS are needed. Uniformity of concepts of PCOS, anxiety and depression in research and collaboration among different centers are strategies that could promote knowledge about this issue. In addition, studies specifically aimed at the investigation of the association between PCOS and anxiety and/or depression in adolescents, adults, and other minority groups could be relevant, as well as the analysis of other potential confounders (hormone therapy, medical treatment for PCOS, multiple drug interaction, etc). In view of the considerable potential of anxiety and depression disorders to impair functional ability, ${ }^{34}$ studies investigating whether treating these conditions could improve the outcome of these patients should be greatly encouraged.

\section{Acknowledgments}

This work was supported by grants from the National Council for Scientific and Technological Development (CNPq; Conselho Nacional de Desenvolvimento Científico e Tecnológico) to SLB, research grant (Bolsista de Produtividade em Pesquisa 305274/2014-7).

\section{Disclosure}

The authors report no conflicts of interest in this work.

\section{References}

1. Dunaif A. Polycystic ovary syndrome in 2011: genes, aging and sleep apnea in polycystic ovary syndrome. Nat Rev Endocrinol. 2012;8(2): 72-74.

2. Ali AT. Polycystic ovary syndrome and metabolic syndrome. Ceska Gynekol. 2015;80(4):279-289.

3. Annagur BB, Kerimoglu OS, Tazegul A, Gunduz S, Gencoglu BB. Psychiatric comorbidity in women with polycystic ovary syndrome. J Obstet Gynaecol Res. 2015;41(8):1229-1233.

4. Dokras A. Mood and anxiety disorders in women with PCOS. Steroids. 2012;77(4):338-341.

5. Davari-Tanha F, Hosseini Rashidi B, Ghajarzadeh M, Noorbala AA. Bipolar disorder in women with polycystic ovarian syndrome (PCO). Acta Med Iran. 2014;52(1):46-48.
6. Kerchner A, Lester W, Stuart SP, Dokras A. Risk of depression and other mental health disorders in women with polycystic ovary syndrome: a longitudinal study. Fertil Steril. 2009;91(1):207-212.

7. Naqvi SH, Moore A, Bevilacqua K, et al. Predictors of depression in women with polycystic ovary syndrome. Arch Womens Ment Health. 2015;18(1):95-101.

8. Blay SL. A case of major depressive disorder and symptoms of polycystic ovary syndrome responding to escitalopram. Prim Care Companion CNS Disord. 2011;13(6).

9. Moher D, Liberati A, Tetzlaff J, Altman DG; PRISMA Group. Preferred reporting items for systematic reviews and meta-analyses: the PRISMA statement. J Clin Epidemiol. 2009;62(10):1006-1012.

10. Higgins JPT, Green S, editors. Cochrane Handbook for Systematic Reviews of Interventions Version 5.0.2. The Cochrane Collaboration; 2008.

11. Rotterdam ESHRE/ASRM-Sponsored PCOS consensus workshop group. Revised 2003 consensus on diagnostic criteria and long-term health risks related to polycystic ovary syndrome (PCOS). Hum Reprod. 2004;19(1):41-47.

12. Wells GA, Shea B, O'Connell D, et al. The Newcastle-Ottawa Scale (NOS) for assessing the quality of nonrandomised studies in metaanalyses. Ottawa, ON: Ottawa Hospital Research Institute; 2011. Available from: http://www.ohri.ca/programs/clinical_epidemiology/ oxford.asp. Accessed September 21, 2016.

13. Viechtbauer W. Conducting Meta-Analyses in $\mathrm{R}$ with the metafor Package. Journal of Statistical Software. 2010;36(3):1-48.

14. Egger M, Davey Smith G, Schneider M, Minder C. Bias in metaanalysis detected by a simple, graphical test. BMJ. 1997;315(7109): 629-634.

15. Higgins JP, Thompson SG. Quantifying heterogeneity in a metaanalysis. Stat Med. 2002;21(11):1539-1558.

16. Van Houwelingen HC, Arends LR, Stijnen T. Advanced methods in meta-analysis: multivariate approach and meta-regression. Stat Med. 2002;21(4):589-624.

17. Thompson SG, Sharp SJ. Explaining heterogeneity in meta-analysis: a comparison of methods. Stat Med. 1999;18(20):2693-2708.

18. Zueff LN, da Silva Lara LA, Vieira CS, Martins Wde P, Ferriani RA. Body composition characteristics predict sexual functioning in obese women with or without PCOS. J Sex Marital Ther. 2015;41(3): 227-237.

19. Asik M, Altinbas K, Eroglu M, et al. Evaluation of affective temperament and anxiety-depression levels of patients with polycystic ovary syndrome. J Affect Dis. 2015;185:214-218.

20. Moran LJ, Deeks AA, Gibson-Helm ME, Teede HJ. Psychological parameters in the reproductive phenotypes of polycystic ovary syndrome. Hum Reprod. 2012;27(7):2082-2088.

21. Moran L, Gibson-Helm M, Teede H, Deeks A. Polycystic ovary syndrome: a biopsychosocial understanding in young women to improve knowledge and treatment options. J Psychosom Obstet Gynaecol. 2010; 31(1):24-31.

22. Sahingöz M, Uguz F, Gezginc K, Korucu DG. Axis I and Axis II diagnoses in women with PCOS. Gen Hosp Psychiatry. 2013;35(5): 508-511.

23. Hollinrake E, Abreu A, Maifeld M, Van Voorhis BJ, Dokras A. Increased risk of depressive disorders in women with polycystic ovary syndrome. Fertil Steril. 2007;87(6):1369-1376.

24. Himelein MJ, Thatcher SS. Depression and body image among women with polycystic ovary syndrome. J Health Psychol. 2006;11(4): 613-625.

25. Marsh CA, Berent-Spillson A, Love T, et al. Functional neuroimaging of emotional processing in women with polycystic ovary syndrome: a case-control pilot study. Fertil Steril. 2013;100(1):200-207.e1.

26. Mueller SC, Ng P, Sinaii N, et al. Psychiatric characterization of children with genetic causes of hyperandrogenism. Eur J Endocrinol. 2010;163(5):801-810.

27. Vreeburg SA, Zitman FG, van Pelt J, et al. Salivary cortisol levels in persons with and without different anxiety disorders. Psychosom Med. 2010;72(4):340-347. 
28. Lanzone A, Petraglia F, Fulghesu AM, Ciampelli M, Caruso A, Mancuso S. Corticotropin-releasing hormone induces an exaggerated response of adrenocorticotropic hormone and cortisol in polycystic ovary syndrome. Fertil Steril. 1995;63(6):1195-1199.

29. Mahmoud R, Wainwright SR, Galea LA. Sex hormones and adult hippocampal neurogenesis: regulation, implications, and potential mechanisms. Front Neuroendocrinol. 2016;41:129-152.

30. Pariante CM, Lightman SL. The HPA axis in major depression: classical theories and new developments. Trends Neurosci. 2008;31(9): 464-468.

31. Macut D, Bozic Antic I, Nestorov J, et al. The influence of combined oral contraceptives containing drospirenone on hypothalamic-pituitaryadrenocortical axis activity and glucocorticoid receptor expression and function in women with polycystic ovary syndrome. Hormones (Athens). 2015;14(1):109-117.
32. Barry JA, Kuczmierczyk AR, Hardiman PJ. Anxiety and depression in polycystic ovary syndrome: a systematic review and meta-analysis. Hum Reprod. 2011;26(9):2442-2451.

33. Veltman-Verhulst SM, Boivin J, Eijkemans MJ, Fauser BJ. Emotional distress is a common risk in women with polycystic ovary syndrome: a systematic review and meta-analysis of 28 studies. Hum Reprod Update. 2012;18(6):638-651.

34. Whiteford HA, Degenhardt L, Rehm J, et al. Global burden of disease attributable to mental and substance use disorders: findings from the Global Burden of Disease Study 2010. Lancet. 2013;382(9904): $1575-1586$.
Neuropsychiatric Disease and Treatment

\section{Publish your work in this journal}

Neuropsychiatric Disease and Treatment is an international, peerreviewed journal of clinical therapeutics and pharmacology focusing on concise rapid reporting of clinical or pre-clinical studies on a range of neuropsychiatric and neurological disorders. This journa is indexed on PubMed Central, the 'PsycINFO' database and CAS,

\section{Dovepress}

and is the official journal of The International Neuropsychiatric Association (INA). The manuscript management system is completely online and includes a very quick and fair peer-review system, which is all easy to use. Visit http://www.dovepress.com/testimonials.php to read real quotes from published authors.

Submit your manuscript here: http://www.dovepress.com/neuropsychiatric-disease-and-treatment-journal 